\title{
The Relationship between Hand Grip Strength and the Accuracy of Field Tennis Services in Eighth Semester Male Students in Department of Training Faculty of Sports Science UNIMA
}

\author{
Ewendi Wenik Mangolo', Fredrik Alfrets Makadada ${ }^{2}$ \\ ${ }^{1}$ Education major, Cenderawasih State University, Jayapura, Indonesia \\ ${ }^{2}$ Department of Sport Science, Manado State University, Indonesia \\ ewendimangolorocketmail.com
}

\begin{abstract}
The aims of this study is to find out the relationship between hand grip strength and the accuracy of field tennis services. In this study the research method is quantitative research with independent variables Hand Grip Strength $\left(X_{I}\right)$, Sleeve Length $\left(X_{2}\right)$ and dependent variable Accuracy of Tennis Service $(Y)$. The result show there is a significant relationship between hand grip strength and service accuracy in semester VII male students of the Department of Coaching Education. There is a meaningful relationship between arm length and correct service in $7^{\text {th }}$ semester male students in Department of Coaching Education. There is a meaningful relationship between hand grip strength and arm length together with the accuracy of service to seventh semester male students of the Department of Training Education.
\end{abstract}

\author{
Keywords \\ Hand grip; accuracy of \\ field tenis; male student
}

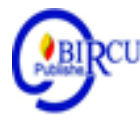

\section{Introduction}

Sports and Health Physical Education is one of the education carried out in Indonesia which has a learning system based on theory and practice. Sports and Health Physical Education is generally taught to students in elementary schools. Sports and Health Physical Education is a learning process through physical activities where each student is obliged to do physical activities to improve his physical fitness. Sports and Health Physical Education not only focuses on improving physical fitness or physical development, but also developing cognitive and affective aspects. (Novianti, 2020)

Tennis is a game that uses arms to swing singly (one on one) and doubles (two against two) and tennis is a complex sport, which uses almost all physical components. The components that are considered important and needed to assess physical ability are strength and accuracy, because basically strength and accuracy are an important component of physical condition in hitting and directing the ball to the right target. Mochamad Sajoto said that: "Muscle strength is a component of physical condition that can be increased to the sub maximal limit, according to the needs of every sport that requires it".

Tennis is a sport that is played by everyone, both men and women of all ages. It can even be done by people with disabilities with special tennis for them, therefore the tennis court has enough fans to deal with it. In the game of tennis also requires a lot of physical skills and abilities, a technique that is a unit that supports each other. Therefore the coach is required to foster and train athletes so as to produce athletes who have potential and achievement.

Many factors affect an athlete's achievement. One of these factors is physical ability, physical ability is not only determined by one factor, but is formed by various factors or components. Physical condition is one of the prerequisites that are needed in every effort to improve an athlete's achievement. Physical conditions are a unified whole of the components 
that cannot be separated, both improvement and maintenance. In the game of tennis there are some basic techniques that must be mastered by every player, therefore mastery of the basic techniques in the game of tennis must have received serious attention early in the effort to develop and improve the game. The basic techniques in pitch tennis include service, forhand, backhand, volleyball, and smash.

\section{Review of Literature}

\subsection{The essence of the accuracy of field tennis services}

Accuracy is one of the physical components that must be possessed by an athlete in the form of sports, especially tennis. Mochamad Sajoto said that: "Accuracy is the ability of a person to control free movements, towards a target. The target can be a distance or maybe a direct object that must be charged ". So service accuracy is the ability to do the right service at the service target.

There are various types of services according to Soediharso consisting of: "Flat or Cannon Ball: the ball is hit with the face of a racket that is perpendicular, Slice: the ball is hit from right to left, and American Twist: the direction of the ball to the left of the opponent". To master these services certainly cannot be separated from exercises that are carried out in a well programmed.

\subsection{The Nature of the Strength of Hand Grips}

The strength of the hand grip can be measured with a hand and grip dynamometer 10 tool that is used to determine the strength of the hand muscles when grasping. A form of physical condition component that is useful for servicing. With good hand grip, tennis rackets can be held tightly without being detached when hitting the ball or receiving a hard ball. Training by using tools that can increase the strength of grip is very important to support sports activities. To enable achievement improvement, training must be guided by certain theories and training principles. Without doing routine exercises, it is impossible for athletes to obtain the expected achievements.

According to Bompa "exercise is a sporting activity which is carried out systematically over a long period of time and is progressively increased and individually leads to the characteristics of physiological and psychological functions to achieve the determined targets". Training has certain limitations, therefore we need to understand what the limits of the practice are. In simple terms the training limit according to Harsono: "Exercise is a systematic process of practicing that is done repeatedly with increasing days increasing the amount of training load".

Although the exercise is carried out repeatedly, systematically and increasingly adds to the burden, besides that the principle of exercise is also important as a guideline for anyone who wants to improve their sports performance. Exercise in principle puts physical pressure on the body regularly and systematically, continuously or continuously so that it will improve the ability of athletes' skills. And to carry out an exercise required appropriate training methods as a condition for achieving goals.

From the description above it can be concluded that training is a planned and systematic activity to prepare athletes from both physical and technical conditions to deal with pressure in comparison. 


\subsection{The Length of the Arm Essence}

The arm is one of the organs of the human body that has an important role in sports activities especially in daily life. Arm length is a human body structure that can be measured from the shoulder (shoulder to shoulder) to the tip of the middle finger. By looking at human body movements, the arm can be divided into two parts, namely the upper arm and lower arm. According to Syaifuddin the muscles of the upper arm consist of: "the biceps brachulus, the brachial muscle, the brachial korako muscle, etc." While the muscles of the forearm consist of: $\mathrm{m}$. extensor carpi radialis longus, m. extensor carpi radialis brevis, and m. ektensor karpi ulnaris, digitorium karpi radialis, m. extensor policis longus, etc.
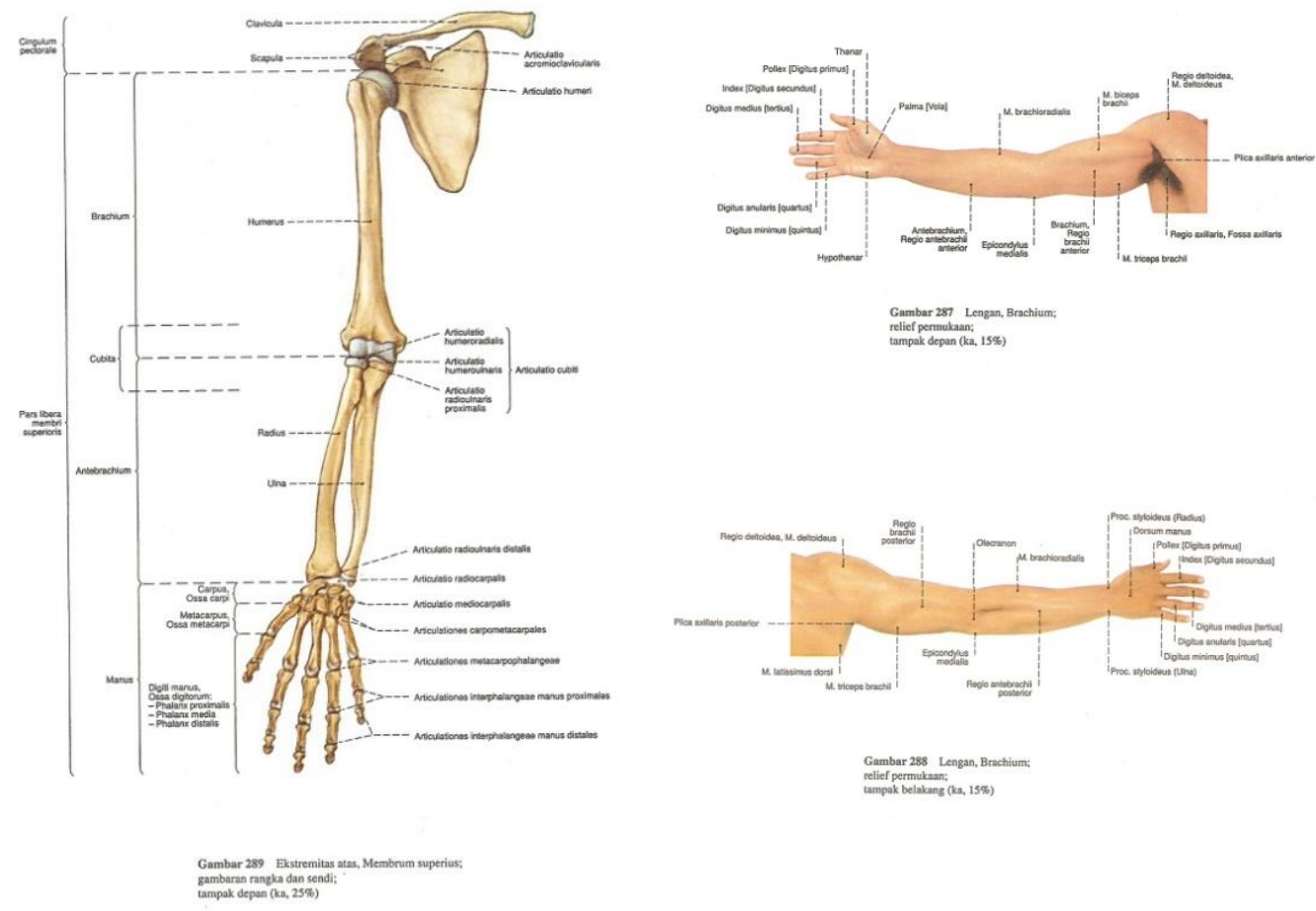

Figure 1. Position of Measurement of Arm Length

As said by Evelin C. Pearce that: "The body frame with the shoulder bracelet consisting of clavicle and scapula, underneath there are bones that form the skeleton of the arm, forearm, palms totaling 30 pieces of bone".

\section{Research Method}

In this study the research variables are:

1. The independent variable: a. Hand Grip Strength $\left(\mathrm{X}_{1}\right)$

b. Sleeve Length $\left(\mathrm{X}_{2}\right)$

2. Dependent variable: Accuracy of Tennis Service (Y)

The strength of the hand grip is one component of the physical conditions needed in tennis. With a good hand grip, it will be easier for athletes to hold the racket firmly and create more efficient blows in relation to the use of the hand muscles. Exercise is very important to help improve the ability to do sports activities. To enable increased achievement, training must be guided by certain theories and training principles. Hand grip strength must be trained 
by providing training in a programmed and continuous or repetitive manner, this form of exercise is in the form of a hand grip device. For example by squeezing the tool with regular exercise. And using a dynamometer hand and grip measuring instrument with interval scale.

Someone's arm length is different from one another. Arm length is one of the supporting factors in athletes doing good service. And everything can be seen from someone's posture or even measured by the $\mathrm{cm}$ unit with an interval scale.

As has been explained that if the tennis player wants his opponent to be unable to return his service and earn points, then the strength of the hand grip and arm length and the accuracy of the service of the player must be properly trained, because if the player has it all will make it easier to get points from his opponent with service that is effective and efficient.

Service accuracy is the ability for someone to do the right service on target and is difficult for the opponent to reach. Accuracy is a very important component in playing tennis on the pitch to aim the ball at the target so that it can complicate the opponent's reach and can generate points without expending excessive energy. "And the ability to measure service accuracy can be measured by the number points specified in the service area or area".

\subsection{Research Design}

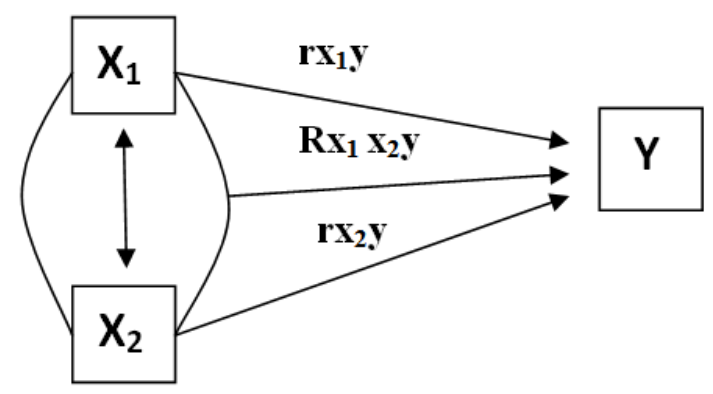

Figure 2. Research Design $\mathrm{X}_{1} \mathrm{X}_{2}$ and $\mathrm{Y}^{2}$

Information :

$\mathrm{X}_{1}=$ Strength of Hand Grips

$\mathrm{X}_{2}=$ Arm Length

$\mathrm{Y}=$ Service Accuracy

\subsection{Place and time of research}

1. Place of Research

UNIMA Tennis Court and UNIMA Laboratory in Tondano

2. Research Time

The time needed to collect research data is 1 (one) week at $14.00-17.00$

According to Arikunto, quoted by Sinaga: "If the subject or population is less than 100 people, all of them are taken so that the research is a population study. Whereas if the number of subjects is more than 100 people, it is taken between $10-15 \%$ or $20-25 \%$ or more

". How to take population and sample taken by using the formula and steps as follows:

1. Population: are all semester VII male students of the FIK-UNIMA Coaching Education Department with 40 students.

2. Sample: is the total population of 40 people. Thus this study is a population study. The instruments of this research are:

1. The strength of the hand grip is used by the hand and grip dynamometer.

2. The length of the arm is used in meters $\mathrm{cm}$. 
3. Service accuracy is used in measuring meters with numbers designed by Hewit, et al in the book "Measurment concepts in Physical Education".
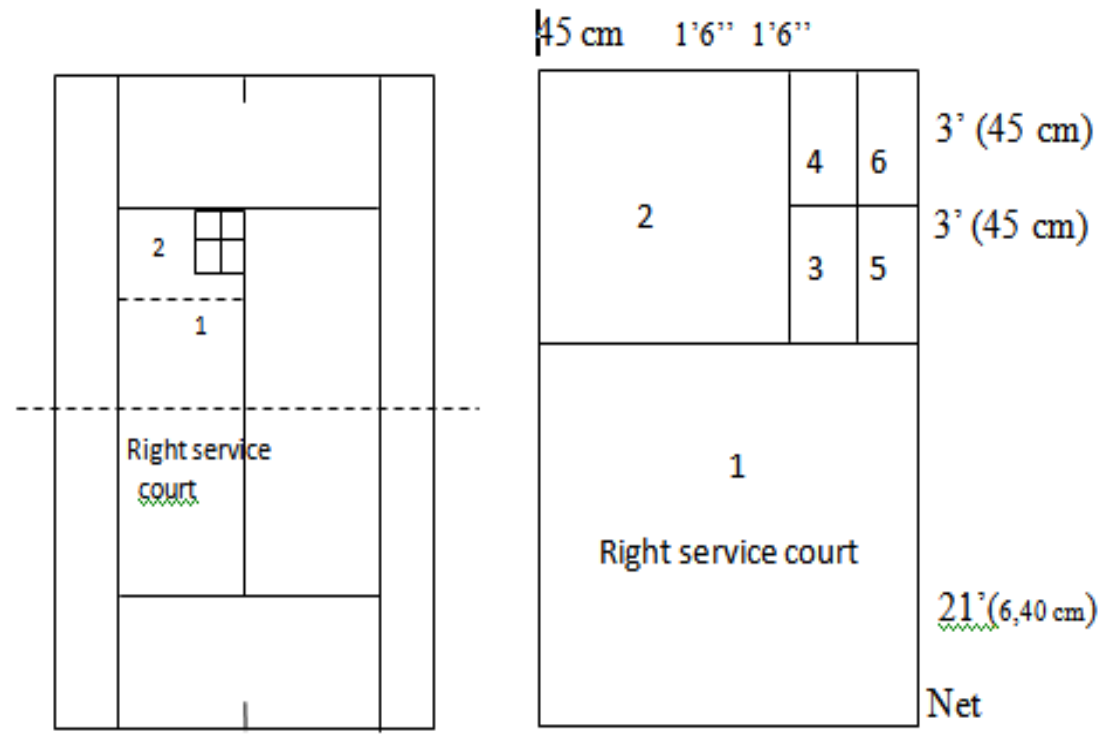

$13^{\prime} 6^{\prime \prime}$

Table 1. Tennis test board, According to Hewit Jack et al.

Subject: Male and Female College

Validity: Correlation Coefficient

\begin{tabular}{|l|c|c|c|}
\cline { 2 - 4 } \multicolumn{1}{c|}{} & Varsity-Junior varsity & Advanced & Beginners \\
\hline Forehand & .57 & .62 & .67 \\
\hline Backhand & .52 & .61 & .62 \\
\hline Serve placement & .93 & .63 & .89 \\
\hline Speed serve & .86 & .72 & \\
\hline
\end{tabular}

Tools and Equipment

- Tennis court

- Tennis balls as much as 30 pcs

- Tennis racket

- Net

- Chalk

- meter gauge

a. Service accuracy is measured by:

Testi stands behind the service line and is ready to serve. Testi are given 10 (ten) service opportunities only in the right position then the service box is divided into six boxes and each box has its own value according to the difficulty level. Each ball that easily passes the net is scored according to the value indicated on the service target box. A ball that is not entered is given a value of 0 . The service accuracy value of each child is the sum of values obtained from the 10 balls that are serviced. By Hewit 
Table 2. Criteria in the test

\begin{tabular}{|c|c|c|}
\hline College Men & Performance Level & College Women \\
\hline $20-60$ & Excellent & $14-60$ \\
\hline $16-19$ & Good & $10-13$ \\
\hline $7-15$ & Average & $4-9$ \\
\hline $3-6$ & Poor & $1-3$ \\
\hline $0-2$ & Very Poor & 0 \\
\hline
\end{tabular}

\section{b. A hand grip test is taken by:}

The male students stood up straight and relaxed then were given the opportunity to use the dynamometer hand and grip 2 (two) times.

c. The arm length test is measured by:

The male student stands, then the arms are stretched on the table and the arms are measured by a meter from the shoulder wrist to the tip of the middle finger.

Data collection on hand grip strength, arm length and service accuracy were carried out in October 2013, with the following schedule:

Table 3. Arm length test

\begin{tabular}{|c|c|c|c|c|}
\hline $\mathrm{c}$ & ACTIVITY & IMPLEMENTATION & $\begin{array}{c}\text { THE } \\
\text { PLACE }\end{array}$ & INF \\
\hline 2. & $\begin{array}{l}\text { Hand grip strength test } \\
\text { Measuring arm length }\end{array}$ & $\begin{array}{l}\text { Monday,15-10-2013 } \\
\text { Time 14.00-Finish } \\
\text { Monday,15-10-2013 } \\
\text { Time14.00-Finish }\end{array}$ & $\begin{array}{l}\text { Lab. FIK, } \\
\text { Lap. Tenis } \\
\text { FIK } \\
\text { Lab. FIK, } \\
\text { Lap. Tenis } \\
\text { FIK }\end{array}$ & $\begin{array}{l}\text { The whole } \\
\text { test is taken } \\
3 \text { times, } \\
\text { because the } \\
\text { time of the } \\
\text { sample to be } \\
\text { tested is } \\
\text { adjusted. }\end{array}$ \\
\hline & & $\begin{array}{l}\text { Wednesday, 17-10- } \\
2013 \\
\text { Jam 14.00-selesai }\end{array}$ & $\begin{array}{l}\text { Lap. Tenis } \\
\text { FIK UNIMA }\end{array}$ & \\
\hline
\end{tabular}

\subsection{Data Analysis Techniques}

Hypothesis 1 and 2 analysis techniques use "Product Moment Correlation Analysis" as follows:

$$
\text { Hypothesis } 1: r_{x y}=\frac{n \sum \mathrm{X} 1 \mathrm{Y}-(\Sigma \mathrm{X} 1)}{\sqrt{\left[( n \sum \mathrm { X } 1 ^ { 2 } - ( \Sigma \mathrm { X } 1 ) ^ { 2 } ] \left[\mathrm{n} \Sigma \mathrm{Y}^{2}-(\Sigma \mathrm{Y})^{2}\right.\right.}}
$$

Information :

$$
\text { Hypothesis } 2: r_{x y}=\frac{n \sum \mathrm{X} 2 \mathrm{Y}-\left(\sum \mathrm{X} 2\right)}{\sqrt{\left[( n \sum \mathrm { X } 2 ^ { 2 } - ( \Sigma \mathrm { X } 2 ) ^ { 2 } ] \left[\mathrm{n} \Sigma \mathrm{Y}^{2}-(\Sigma \mathrm{Y})^{2}\right.\right.}}
$$

$$
\begin{aligned}
& \mathrm{r}_{\mathrm{xy}}=\text { correlation coefficient } \\
& \mathrm{n}=\text { Number of samples } \\
& \quad \sum \mathrm{XY}=\text { Number of times } \mathrm{x} \text { and } \mathrm{y} \\
& \sum \mathrm{X}=\text { Total } \mathrm{x} \\
& \sum \mathrm{Y}=\text { Number of } \mathrm{y} \\
& \sum \mathrm{X}_{1}=\text { Number of values for variable } \mathrm{X}_{1} \\
& \sum \mathrm{X}_{1}{ }^{2}=\text { Number of values of variable } \mathrm{X}_{1} \text { squared } \\
& \sum \mathrm{Y}^{2}=\text { Total value of } \mathrm{y} \text { squared }
\end{aligned}
$$


For the third hypothesis analysis using multiple correlation analysis (Multiple Correlation) as follows:

$R y\left(x_{1,} x_{2}\right)=\sqrt{\frac{\sum \mathrm{r}^{2} \mathrm{yx} 1+\mathrm{r}^{2} \mathrm{yx} 2-2 . \mathrm{ry} \times 1 . \mathrm{ry} \times 2 \cdot \mathrm{rrx} 1 \times 2}{1-\mathrm{r}^{2} \mathrm{x} 1 \times 2}}$

Information:

Ryx1x2 = Double correlation coefficient between $\mathrm{X}_{1}$ and $\mathrm{X}_{2}$ with $\mathrm{Y}$

ryx $1=$ correlation coefficient $\mathrm{X}_{1}$ with $\mathrm{Y}$

ryx 2 = Correlation coefficient $\mathrm{X}_{2}$ with $\mathrm{Y}$

$\mathrm{rx} 1 \times 2=$ Correlation coefficient $\mathrm{X}_{1}$ with $\mathrm{X}_{2}$

To start the analysis, it is preceded by a test of requirements, namely a test for normality and linearity.

\section{Discussion}

The data analyzed were variable $\mathrm{X}_{1}$ (hand grip strength), variable $\mathrm{X}_{2}$ (arm length) and $\mathrm{Y}$ variable (service accuracy). The research data can be seen in table 1 as follows:

Table 4. Research Results of Hand Grip Strength $\left(X_{1}\right)$, Arm Length $\left(X_{2}\right)$ and Service Accuracy (Y)

\begin{tabular}{|c|c|c|c|}
\hline No & $\begin{array}{c}\text { Strength of Hand } \\
\text { Grips } \\
\left(\mathbf{X}_{\mathbf{1}}\right)\end{array}$ & $\begin{array}{c}\text { Sleeve Length } \\
\left.\mathbf{( X}_{\mathbf{2}}\right)\end{array}$ & $\begin{array}{c}\text { Service } \\
\text { Accuracy } \\
(\mathbf{Y})\end{array}$ \\
\hline 1 & 37 & 58 & 37 \\
\hline 2 & 47 & 61 & 33 \\
\hline 3 & 45 & 63 & 35 \\
\hline 4 & 39 & 66 & 39 \\
\hline 5 & 39 & 57 & 32 \\
\hline 6 & 27 & 59 & 27 \\
\hline 7 & 48 & 66 & 48 \\
\hline 8 & 43 & 69 & 43 \\
\hline 9 & 36 & 62 & 48 \\
\hline 10 & 31 & 65 & 31 \\
\hline 11 & 46 & 70 & 43 \\
\hline 12 & 25 & 54 & 27 \\
\hline 13 & 47 & 69 & 47 \\
\hline 14 & 44 & 67 & 44 \\
\hline 15 & 31 & 57 & 31 \\
\hline 16 & 24 & 52 & 24 \\
\hline 17 & 35 & 58 & 32 \\
\hline 18 & 36 & 64 & 38 \\
\hline 19 & 43 & 65 & 43 \\
\hline 20 & 31 & 59 & 31 \\
\hline 21 & 37 & 61 & 37 \\
\hline 22 & 43 & 60 & 45 \\
\hline 23 & 27 & 58 & 29 \\
\hline 24 & 31 & 59 & 31 \\
\hline 25 & 42 & 65 & 46 \\
\hline 26 & 43 & & 45 \\
\hline
\end{tabular}




\begin{tabular}{|l|l|l|l|}
\hline 27 & 35 & 63 & 40 \\
\hline 28 & 34 & 66 & 34 \\
\hline 29 & 39 & 79 & 39 \\
\hline 30 & 43 & 65 & 34 \\
\hline 31 & 44 & 67 & 36 \\
\hline 32 & 45 & 63 & 34 \\
\hline 33 & 47 & 69 & 38 \\
\hline 34 & 43 & 65 & 37 \\
\hline 35 & 46 & 74 & 46 \\
\hline 36 & 49 & 70 & 43 \\
\hline 37 & 47 & 68 & 37 \\
\hline 38 & 36 & 78 & 43 \\
\hline 39 & 53 & 68 & 48 \\
\hline 40 & 34 & 70 & 39 \\
\hline
\end{tabular}

From the results of measurements in table 1, then assisted by using the Casio fx-85ms Calculator obtained prices needed for further analysis, both on variable X1, X2 variable and Y variable, as follows:
$\mathrm{n}=40$
$\sum \mathrm{X}_{1}^{2}=63816$
$\mathrm{Sdx}_{1}=7.23$
$\sum \mathrm{X}_{1}=1572$
$\sum \mathrm{X}_{2}^{2}=168674$
$\mathrm{Sdx}_{2}=6.18$
$\sum \mathrm{X}_{2}=2586$
$\sum \mathrm{Y}^{2}=58956$
Sdy $=6.51$
$\sum \mathrm{Y}=1514$
$\sum \mathrm{X}_{1} . \mathrm{Y}=60793$
$\mathrm{Sdx}_{1}^{2}=52.2729$
$\overline{\mathrm{X}}_{1}=39.3$
$\sum X_{2} . Y=98904$
$\mathrm{Sdx}_{2}^{2}=38.1924$
$\overline{\mathrm{X}}_{2}=64.65$
$\sum \mathrm{X}_{1} \cdot \mathrm{X}_{2}=102595$
$\mathrm{Sdy}^{2}=42.3801$
$\overline{\mathrm{Y}}=37.85$

The results of research on the strength of hand grip $\left(\mathrm{X}_{1}\right)$ and arm length $\left(\mathrm{X}_{2}\right)$ with service accuracy $(\mathrm{Y})$ after changing raw scores into standard scores or raising ordinal data into interval data using the $\mathrm{T}$ scale with the formula:

$$
\text { T-Score }=50+10\left(\frac{x-\bar{x}}{s d}\right)
$$

Then the T score results are as follows:

Table 5. Raw Data Becomes Raw Data (Increasing Ordinal Data to Interval)

\begin{tabular}{|c|c|c|c|c|c|c|}
\hline \multirow{2}{*}{ NO } & \multicolumn{2}{|c|}{ CRUDE SCORE (CRUDE DATA) } & \multicolumn{3}{|c|}{ RAW SCORE RESULTS } \\
\cline { 2 - 7 } & $\mathbf{X}_{\mathbf{1}}$ & $\mathbf{X}_{\mathbf{2}}$ & $\mathbf{Y}$ & $\mathbf{X}_{\mathbf{1}}$ & $\mathbf{X}_{\mathbf{2}}$ & $\mathbf{Y}$ \\
\hline $\mathbf{1}$ & 37 & 58 & 37 & 47 & 39 & 49 \\
\hline $\mathbf{2}$ & 47 & 61 & 33 & 61 & 44 & 43 \\
\hline $\mathbf{3}$ & 45 & 63 & 35 & 58 & 47 & 46 \\
\hline $\mathbf{4}$ & 39 & 66 & 39 & 50 & 52 & 52 \\
\hline $\mathbf{5}$ & 39 & 57 & 32 & 50 & 38 & 41 \\
\hline $\mathbf{6}$ & 27 & 59 & 27 & 33 & 41 & 33 \\
\hline $\mathbf{7}$ & 48 & 66 & 48 & 62 & 52 & 66 \\
\hline $\mathbf{8}$ & 43 & 69 & 43 & 55 & 57 & 58 \\
\hline $\mathbf{9}$ & 36 & 62 & 48 & 45 & 46 & 66 \\
\hline
\end{tabular}




\begin{tabular}{|c|c|c|c|c|c|c|}
\hline 10 & 31 & 65 & 31 & 39 & 51 & 40 \\
\hline 11 & 46 & 70 & 43 & 59 & 59 & 58 \\
\hline 12 & 25 & 54 & 27 & 30 & 33 & 33 \\
\hline 13 & 47 & 69 & 47 & 61 & 57 & 64 \\
\hline 14 & 44 & 67 & 44 & 57 & 54 & 59 \\
\hline 15 & 31 & 57 & 31 & 39 & 38 & 40 \\
\hline 16 & 24 & 52 & 24 & 29 & 30 & 29 \\
\hline 17 & 35 & 58 & 32 & 44 & 39 & 41 \\
\hline 18 & 36 & 64 & 38 & 45 & 49 & 50 \\
\hline 19 & 43 & 65 & 43 & 55 & 51 & 58 \\
\hline 20 & 31 & 59 & 31 & 39 & 41 & 40 \\
\hline 21 & 37 & 61 & 37 & 47 & 44 & 49 \\
\hline 22 & 43 & 60 & 45 & 55 & 43 & 61 \\
\hline 23 & 27 & 58 & 29 & 33 & 39 & 36 \\
\hline 24 & 31 & 59 & 31 & 39 & 41 & 40 \\
\hline 25 & 42 & 65 & 46 & 54 & 51 & 63 \\
\hline 26 & 43 & 77 & 45 & 55 & 70 & 61 \\
\hline 27 & 35 & 63 & 40 & 44 & 47 & 53 \\
\hline 28 & 34 & 66 & 34 & 43 & 52 & 44 \\
\hline 29 & 39 & 79 & 39 & 50 & 73 & 52 \\
\hline 30 & 43 & 65 & 34 & 55 & 51 & 44 \\
\hline 31 & 44 & 67 & 36 & 57 & 54 & 47 \\
\hline 32 & 45 & 63 & 34 & 58 & 47 & 44 \\
\hline 33 & 47 & 69 & 38 & 61 & 57 & 50 \\
\hline 34 & 43 & 65 & 37 & 55 & 51 & 49 \\
\hline 35 & 46 & 74 & 46 & 59 & 65 & 63 \\
\hline 36 & 49 & 70 & 43 & 63 & 59 & 58 \\
\hline 37 & 47 & 68 & 37 & 61 & 55 & 49 \\
\hline 38 & 36 & 78 & 43 & 45 & 72 & 58 \\
\hline 39 & 53 & 68 & 48 & 69 & 55 & 66 \\
\hline 40 & 34 & 70 & 39 & 43 & 59 & 52 \\
\hline
\end{tabular}

By using the $\mathrm{fx}-85 \mathrm{~ms}$ calculator program, we obtain the standard score statistical values for variable data $\mathrm{X}_{1}$ (hand grip strength) and $\mathrm{X}_{2}$ (arm length) with $\mathrm{Y}$ variable (service accuracy) as follows:
$\mathrm{n}=40$
$\sum \mathrm{X}_{1}^{2}=104278$
$\mathrm{Sdx}_{1}=9.97$
$\sum X_{1}=2004$
$\sum \mathrm{X}_{2}{ }^{2}=104175$
$\mathrm{Sdx}_{2}=9.97$
$\sum \mathrm{X}_{2}=2003$
$\sum \mathrm{Y}^{2}=104429$
Sdy $=10.04$
$\sum \mathrm{Y}=2005$
$\sum \mathrm{X}_{1} . \mathrm{Y}=103172$
$\mathrm{Sdx}_{1}{ }^{2}=99.4009$
$\overline{\mathrm{X}}_{1}=50.1$
$\sum X_{2} . Y=102942$
$\mathrm{Sdx}_{2}^{2}=99.4009$
$\overline{\mathrm{X}}_{2}=50.08$
$\sum \mathrm{X}_{1} \cdot \mathrm{X}_{2}=102464$
$\mathrm{Sdy}^{2}=100.8016$
$\mathrm{Y}=50.13$

\subsection{Data Analysis}

Data analysis technique used for the purposes of testing hypotheses in this study is to use product moment correlation followed by multiple correlations that aim to test the research 
hypothesis, before the double correlation is carried out, it begins with testing the analysis requirements, namely normality test data using Lilliefors test and test homogeneity by using large variance test versus small variance.

a. Testing Requirements Analysis

1). Data Normality Testing

\section{- Normality Strength Hand Testing Data}

To find out if the sample comes from a normally distributed population, then the data normality test is performed with the Lilliefors test. The normality test is carried out with the following steps:

a). Step One: Determine the Testing Hypothesis

The testing hypothesis is:

Ho : samples come from populations that are normally distributed

$\mathrm{H}_{\mathrm{A}}$ : the sample comes from populations that are not normally distributed

b). Step Two: Determine Testing Criteria

Testing criteria are:

Terima Ho jika Lo $\leq \mathrm{Lt}$

Accept Ho If $\mathrm{Lo}>\mathrm{Lt}$

Significance level $\alpha 0,05$

c). Third step: calculate $\mathrm{Zi}, \mathrm{F}(\mathrm{Zi}), \mathrm{S}(\mathrm{Zi})$ and the difference in $\mathrm{F}(\mathrm{Zi})-\mathrm{S}(\mathrm{Zi})$ and input them into the table.

Dik: $\quad \bar{x}_{1}=50.1$

$\mathrm{Sdx}_{1}=9.97$

Table 6. Calculation of Normality of Hand Grip Strength Data

\begin{tabular}{|c|c|c|c|c|c|}
\hline $\mathbf{N o}$ & $\mathbf{X}_{\mathbf{1}}$ & $\mathbf{Z}_{\mathbf{i}}$ & $\mathbf{F}\left(\mathbf{Z}_{\mathbf{i}}\right)$ & $\mathbf{S}\left(\mathbf{Z}_{\mathbf{i}}\right)$ & $\mathbf{F}\left(\mathbf{Z}_{\mathbf{i}}\right)-\mathbf{S}\left(\mathbf{Z}_{\mathbf{i}}\right)$ \\
\hline 1 & 29 & -2.12 & 0.0170 & 0.0250 & 0.0080 \\
\hline 2 & 30 & -2.02 & 0.0217 & 0.0500 & 0.0283 \\
\hline 3 & 33 & -1.72 & 0.0427 & 0.1000 & 0.0573 \\
\hline 4 & 33 & -1.72 & 0.0427 & 0.1000 & 0.0573 \\
\hline 5 & 39 & -1.11 & 0.1335 & 0.2000 & 0.0665 \\
\hline 6 & 39 & -1.11 & 0.1335 & 0.2000 & 0.0665 \\
\hline 7 & 39 & -1.11 & 0.1335 & 0.2000 & 0.0665 \\
\hline 8 & 39 & -1.11 & 0.1335 & 0.2000 & 0.0665 \\
\hline 9 & 43 & -0.72 & 0.2358 & 0.2500 & 0.0142 \\
\hline 10 & 43 & -0.72 & 0.2358 & 0.2500 & 0.0142 \\
\hline 11 & 44 & -0.61 & 0.2709 & 0.3000 & 0.0291 \\
\hline 12 & 44 & -0.61 & 0.2709 & 0.3000 & 0.0291 \\
\hline 13 & 45 & -0.51 & 0.3050 & 0.3750 & 0.0700 \\
\hline 14 & 45 & -0.51 & 0.3050 & 0.3750 & 0.0700 \\
\hline 15 & 45 & -0.51 & 0.3050 & 0.3750 & 0.0700 \\
\hline 16 & 47 & -0.31 & 0.3783 & 0.4250 & 0.0467 \\
\hline 17 & 47 & -0.31 & 0.3783 & 0.4250 & 0.0467 \\
\hline 18 & 50 & -0.01 & 0.4960 & 0.5000 & 0.0040 \\
\hline 19 & 50 & -0.01 & 0.4960 & 0.5000 & 0.0040 \\
\hline 20 & 50 & -0.01 & 0.4960 & 0.5000 & 0.0040 \\
\hline 21 & 54 & 0.39 & 0.6517 & 0.5250 & $\mathbf{0 . 1 2 6 7}$ \\
\hline 22 & 55 & 0.49 & 0.6879 & 0.6750 & 0.0129 \\
\hline
\end{tabular}




\begin{tabular}{|l|l|l|l|l|l|}
\hline 23 & 55 & 0.49 & 0.6879 & 0.6750 & 0.0129 \\
\hline 24 & 55 & 0.49 & 0.6879 & 0.6750 & 0.0129 \\
\hline 25 & 55 & 0.49 & 0.6879 & 0.6750 & 0.0129 \\
\hline 26 & 55 & 0.49 & 0.6879 & 0.6750 & 0.0129 \\
\hline 27 & 55 & 0.49 & 0.6879 & 0.6750 & 0.0129 \\
\hline 28 & 57 & 0.69 & 0.7549 & 0.7250 & 0.0299 \\
\hline 29 & 57 & 0.69 & 0.7549 & 0.7250 & 0.0299 \\
\hline 30 & 58 & 0.79 & 0.7852 & 0.7750 & 0.0102 \\
\hline 31 & 58 & 0.79 & 0.7852 & 0.7750 & 0.0102 \\
\hline 32 & 59 & 0.89 & 0.8133 & 0.8250 & 0.0117 \\
\hline 33 & 59 & 0.89 & 0.8133 & 0.8250 & 0.0117 \\
\hline 34 & 61 & 1.09 & 0.8621 & 0.9250 & 0.0629 \\
\hline 35 & 61 & 1.09 & 0.8621 & 0.9250 & 0.0629 \\
\hline 36 & 61 & 1.09 & 0.8621 & 0.9250 & 0.0629 \\
\hline 37 & 61 & 1.09 & 0.8621 & 0.9250 & 0.0629 \\
\hline 38 & 62 & 1.19 & 0.8830 & 0.9500 & 0.0670 \\
\hline 39 & 63 & 1.29 & 0.9015 & 0.9750 & 0.0735 \\
\hline 40 & 69 & 1.90 & 0.9713 & 1.0000 & 0.0287 \\
\hline
\end{tabular}

d). Step four: Summarize the Calculation Results

From the calculations listed in table 2, the highest difference is obtained or Observation value of 0.1267 . Based on the table of critical values Lilliefors Luji at $\square$ 0.05 with $n=40$ found Ltabel worth 0.140. So observations are smaller than Ltable ie $\mathrm{Lo}=0.1267<\mathrm{Lt}=0.140$. Based on the testing criteria if Lo $<\mathrm{Lt}, \mathrm{HO}$ is accepted. Thus the conclusion of the test is that the study sample comes from a normally distributed population.

\section{- Testing Arm Length Normality Data}

To find out whether the sample comes from a normally distributed population, then the data normality test is done with the test

a). The first step: determine the testing hypothesis

The testing hypothesis is:

HO: Samples come from populations that are normally distributed

Ha: Samples come from populations that are not normally distributed.

b). Second step: Determine the testing criteria

Testing criteria are:

Thank Ho if Lo $<\mathrm{Lt}$

Reject Ho if $\mathrm{Lo}>\mathrm{Lt}$

Significance rate $\alpha 0.05$

c). Third step: calculate $\mathrm{Zi}, \mathrm{F}(\mathrm{Zi}), \mathrm{S}(\mathrm{Zi})$ and the difference between $\mathrm{F}(\mathrm{Zi})-\mathrm{S}(\mathrm{Zi})$ and input them into the table.

Dik : $\quad \bar{X}_{2}: 50.08$

$\mathrm{Sdx}_{2} \quad: 9.97$

The product of this research is in the form of developing the steps of a problem based learning model so as to produce a problem based learning model with nine steps in learning to write an exposition text. The development of the learning steps is done by modifying the phases contained in the problem based learning model, as revealed by Hosnan (2014: 295) contained in table 7: 
Table 7. Calculation of Normality Test for Arm Length Data

\begin{tabular}{|c|c|c|c|c|c|}
\hline $\mathbf{N o}$ & $\mathbf{X}_{\mathbf{2}}$ & $\mathbf{Z}_{\mathbf{i}}$ & $\mathbf{F}\left(\mathbf{Z}_{\mathbf{i}}\right)$ & $\mathbf{S}\left(\mathbf{Z}_{\mathbf{i}}\right)$ & $\mathbf{F}\left(\mathbf{Z}_{\mathbf{i}}\right) \mathbf{-} \mathbf{S}\left(\mathbf{Z}_{\mathbf{i}}\right)$ \\
\hline 1 & 30 & -2.01 & 0.0222 & 0.0250 & 0.0028 \\
\hline 2 & 33 & -1.71 & 0.0436 & 0.0500 & 0.0064 \\
\hline 3 & 38 & -1.21 & 0.1131 & 0.1000 & 0.0131 \\
\hline 4 & 38 & -1.21 & 0.1131 & 0.1000 & 0.0131 \\
\hline 5 & 39 & -1.11 & 0.1335 & 0.1750 & 0.0415 \\
\hline 6 & 39 & -1.11 & 0.1335 & 0.1750 & 0.0415 \\
\hline 7 & 39 & -1.11 & 0.1335 & 0.1750 & 0.0415 \\
\hline 8 & 41 & -0.91 & 0.1814 & 0.2500 & 0.0686 \\
\hline 9 & 41 & -0.91 & 0.1814 & 0.2500 & 0.0686 \\
\hline 10 & 41 & -0.91 & 0.1814 & 0.2500 & 0.0686 \\
\hline 11 & 43 & -0.71 & 0.2389 & 0.2750 & 0.0361 \\
\hline 12 & 44 & -0.61 & 0.2709 & 0.3250 & 0.0541 \\
\hline 13 & 44 & -0.61 & 0.2709 & 0.3250 & 0.0541 \\
\hline 14 & 46 & -0.41 & 0.3409 & 0.3500 & 0.0091 \\
\hline 15 & 47 & -0.31 & 0.3783 & 0.4250 & 0.0467 \\
\hline 16 & 47 & -0.31 & 0.3783 & 0.4250 & 0.0467 \\
\hline 17 & 47 & -0.31 & 0.3783 & 0.4250 & 0.0467 \\
\hline 18 & 49 & -0.11 & 0.4562 & 0.4500 & 0.0062 \\
\hline 19 & 51 & 0.09 & 0.5359 & 0.5750 & 0.0391 \\
\hline 20 & 51 & 0.09 & 0.5359 & 0.5750 & 0.0391 \\
\hline 21 & 51 & 0.09 & 0.5359 & 0.5750 & 0.0391 \\
\hline 22 & 51 & 0.09 & 0.5359 & 0.5750 & 0.0391 \\
\hline 23 & 51 & 0.09 & 0.5359 & 0.5750 & 0.0391 \\
\hline 24 & 52 & 0.19 & 0.5753 & 0.6500 & 0.0747 \\
\hline 25 & 52 & 0.19 & 0.5753 & 0.6500 & 0.0747 \\
\hline 26 & 52 & 0.19 & 0.5753 & 0.6500 & 0.0747 \\
\hline 27 & 54 & 0.39 & 0.6517 & 0.7000 & 0.0483 \\
\hline 28 & 54 & 0.39 & 0.6517 & 0.7000 & 0.0483 \\
\hline 29 & 55 & 0.49 & 0.6879 & 0.7500 & 0.0621 \\
\hline 30 & 55 & 0.49 & 0.6879 & 0.7500 & 0.0621 \\
\hline 31 & 57 & 0.69 & 0.7549 & 0.8250 & 0.0701 \\
\hline 32 & 57 & 0.69 & 0.7549 & 0.8250 & 0.0701 \\
\hline 33 & 57 & 0.69 & 0.7549 & 0.8250 & 0.0701 \\
\hline 34 & 59 & 0.89 & 0.8133 & 0.9000 & $\mathbf{0 . 0 8 6 7}$ \\
\hline 35 & 59 & 0.89 & 0.8133 & 0.9000 & $\mathbf{0 . 0 8 6 7}$ \\
\hline 36 & 59 & 0.89 & 0.8133 & 0.9000 & $\mathbf{0 . 0 8 6 7}$ \\
\hline 37 & 65 & 1.50 & 0.9332 & 0.9250 & 0.0083 \\
\hline 38 & 70 & 2.00 & 0.9772 & 0.9500 & 0.0272 \\
\hline 39 & 72 & 2.20 & 0.9861 & 0.9750 & 0.0111 \\
\hline 40 & 73 & 2.30 & 0.9893 & 1.0000 & 0.0107 \\
\hline & & & & & \\
\hline
\end{tabular}

d). Step four: Summarize the calculation results

From the calculations listed in table 3, the highest difference is obtained or Observation value of 0.0867 . Based on the table of critical values of the Lilliefors test at $\square 0.05$ with $n$ $=40$, it was found that the Ltable was worth 0.140. So observations are smaller than Ltable ie $\mathrm{Lo}=0.0867<\mathrm{Lt}=0.140$. Based on the testing criteria if $\mathrm{Lo}<\mathrm{Lt}$, HO is accepted. 
Thus the conclusion of the test is that the study sample comes from a normally distributed population.

\section{- Testing the Normality of Service Accuracy Data}

To find out if the sample comes from a normally distributed population, then the data normality test is done with the lilliefors test. Data normality testing is carried out with the following steps:

a. The first step: determine the testing hypothesis

The testing hypothesis is:

HO: Samples come from populations that are normally distributed

HA: Samples come from populations that are not normally distributed.

b. Second step: Determine the testing criteria

Testing criteria are:

Thank Ho if Lo $\square \mathrm{Lt}$

Reject Ho if Lo> Lt

Significance rate $\mathrm{x} 0.05$

c. Third step: calculate $\mathrm{Zi}, \mathrm{F}(\mathrm{Zi}), \mathrm{S}(\mathrm{Zi})$ and the difference between $\mathrm{F}(\mathrm{Zi})-\mathrm{S}(\mathrm{Zi})$ and input them into the table.

Dik: $=50.13$

sdy $=10.04$

Table 8. Calculation of Data Service Normality Test Accuracy

\begin{tabular}{|c|c|c|c|c|c|}
\hline $\mathbf{N o}$ & $\mathbf{Y}$ & $\mathbf{\mathbf { Z } _ { \mathbf { i } }}$ & $\mathbf{F}\left(\mathbf{Z}_{\mathbf{i}}\right)$ & $\mathbf{S}\left(\mathbf{Z}_{\mathbf{i}}\right)$ & $\mathbf{F}\left(\mathbf{Z}_{\mathbf{i}}\right)-\mathbf{S}\left(\mathbf{Z}_{\mathbf{i}}\right)$ \\
\hline 1 & 29 & -2.10 & 0.0179 & 0.0250 & 0.0071 \\
\hline 2 & 33 & -1.71 & 0.0436 & 0.0750 & 0.0314 \\
\hline 3 & 33 & -1.71 & 0.0436 & 0.0750 & 0.0314 \\
\hline 4 & 36 & -1.41 & 0.0793 & 0.1000 & 0.0207 \\
\hline 5 & 40 & -1.01 & 0.1562 & 0.2000 & 0.0438 \\
\hline 6 & 40 & -1.01 & 0.1562 & 0.2000 & 0.0438 \\
\hline 7 & 40 & -1.01 & 0.1562 & 0.2000 & 0.0438 \\
\hline 8 & 40 & -1.01 & 0.1562 & 0.2000 & 0.0438 \\
\hline 9 & 41 & -0.91 & 0.1814 & 0.2500 & 0.0686 \\
\hline 10 & 41 & -0.91 & 0.1814 & 0.2500 & 0.0686 \\
\hline 11 & 43 & -0.71 & 0.2389 & 0.2750 & 0.0361 \\
\hline 12 & 44 & -0.61 & 0.2709 & 0.3500 & $\mathbf{0 . 0 7 9 1}$ \\
\hline 13 & 44 & -0.61 & 0.2709 & 0.3500 & $\mathbf{0 . 0 7 9 1}$ \\
\hline 14 & 44 & -0.61 & 0.2709 & 0.3500 & $\mathbf{0 . 0 7 9 1}$ \\
\hline 15 & 46 & -0.41 & 0.3409 & 0.3750 & 0.0341 \\
\hline 16 & 47 & -0.31 & 0.3783 & 0.4000 & 0.0217 \\
\hline 17 & 49 & -0.11 & 0.4562 & 0.5000 & 0.0438 \\
\hline 18 & 49 & -0.11 & 0.4562 & 0.5000 & 0.0438 \\
\hline 19 & 49 & -0.11 & 0.4562 & 0.5000 & 0.0438 \\
\hline 20 & 49 & -0.11 & 0.4562 & 0.5000 & 0.0438 \\
\hline 21 & 50 & -0.01 & 0.4960 & 0.5500 & 0.0540 \\
\hline 22 & 50 & -0.01 & 0.4960 & 0.5500 & 0.0540 \\
\hline 23 & 52 & 0.19 & 0.5753 & 0.6250 & 0.0497 \\
\hline 24 & 52 & 0.19 & 0.5753 & 0.6250 & 0.0497 \\
\hline 25 & 52 & 0.19 & 0.5753 & 0.6250 & 0.0497 \\
\hline 26 & 53 & 0.29 & 0.6141 & 0.6500 & 0.0359 \\
\hline
\end{tabular}




\begin{tabular}{|l|l|l|l|l|l|}
\hline 27 & 58 & 0.78 & 0.7823 & 0.7750 & 0.0073 \\
\hline 28 & 58 & 0.78 & 0.7823 & 0.7750 & 0.0073 \\
\hline 29 & 58 & 0.78 & 0.7823 & 0.7750 & 0.0073 \\
\hline 30 & 58 & 0.78 & 0.7823 & 0.7750 & 0.0073 \\
\hline 31 & 58 & 0.78 & 0.7823 & 0.7750 & 0.0073 \\
\hline 32 & 59 & 0.88 & 0.8106 & 0.8000 & 0.0106 \\
\hline 33 & 61 & 1.08 & 0.8599 & 0.8500 & 0.0099 \\
\hline 34 & 61 & 1.08 & 0.8599 & 0.8500 & 0.0099 \\
\hline 35 & 63 & 1.28 & 0.8997 & 0.9000 & 0.0003 \\
\hline 36 & 63 & 1.28 & 0.8997 & 0.9000 & 0.0003 \\
\hline 37 & 64 & 1.38 & 0.9162 & 0.9250 & 0.0088 \\
\hline 38 & 66 & 1.58 & 0.9429 & 1.0000 & 0.0571 \\
\hline 39 & 66 & 1.58 & 0.9429 & 1.0000 & 0.0571 \\
\hline 40 & 66 & 1.58 & 0.9429 & 1.0000 & 0.0571 \\
\hline
\end{tabular}

d. Step four: conclude the calculation results

From the calculations listed in table 4, the highest difference or observation is 0.0791 . Based on the table of critical values Lilliefors Luji at $\square 0.05$ with $n=30$ found Ltabel worth 0.140. So observations are smaller than Ltable ie Lo $=0.0791<\mathrm{Lt}=0.140$. Based on the testing criteria if Lo $<\mathrm{Lt}, \mathrm{HO}$ is accepted. Thus the conclusion of testing.

e. Homogeneity Variance Testing

The purpose of testing the variance homogeneity in this study is to determine whether the variance of the study population is homogeneous or not. To find out whether the population variance is homogeneous, then homogeneity testing is done with the largest variance compared to the smallest variance.

Table 9. Test the Variance Homogeneity with the Biggest Variance than the Smallest

\begin{tabular}{|c|c|c|c|}
\hline \multicolumn{4}{|c|}{ Variance } \\
\hline \multirow{2}{*}{$\begin{array}{c}\text { Sample } \\
\text { Variance } \\
\text { Value }\end{array}$} & \multicolumn{3}{|c|}{ Variable Type } \\
\hline & $\begin{array}{c}\text { Strength of Hand } \\
\text { Grips } \\
\left(\mathbf{X}_{1}\right)\end{array}$ & $\begin{array}{c}\text { Sleeve } \\
\text { Length } \\
\left(\mathbf{X}_{2}\right)\end{array}$ & $\begin{array}{c}\text { Service } \\
\text { Accuracy } \\
\text { (Y) }\end{array}$ \\
\hline$S$ & 99.4009 & 99.4009 & 100.8016 \\
\hline $\mathrm{N}$ & 40 & 40 & 40 \\
\hline
\end{tabular}

The steps for testing the variance homogeneity by using a large variance homogeneity test compared to a small variance are as follows:

a. The first step: look for the value of large variance and small variance with the formula:

$\mathrm{F}=\frac{\text { Varians Besar }}{\text { Varians Kecil }}=\frac{100.8016}{99.4009}=1.01$

b. Second step: compare the calculated $F$ value with $F$ table with the formula:

$\mathrm{dk}$ numerator $=\mathrm{n}-1=40-1=39$ (for large variances)

$\mathrm{d}$ denominator $=\mathrm{n}-1=39-1=38$ (for small variances)

significance level $\square 0.05$, then look at table $F$ to get $F$ table $=1.72$ (interpolation).

(Appendix 10)

With the following testing criteria: 
If $\mathrm{F}$ arithmetic $\geq \mathrm{F}$ table means not homogeneous and $\mathrm{F}$ count $\leq \mathrm{F}$ table means homogeneous. It turns out that $\mathrm{F}$ arithmetic $=\mathrm{F}$ table or $1.01<1.72$, then the variances are homogeneous.

Furthermore, to test and prove the hypothesis that has been proposed can be used moment correlation Products test statistics for the first hypothesis (X1 analysis with Y or the relationship between hand grip strength with service accuracy) and the second hypothesis $(\mathrm{X} \neg 2$ analysis with $\mathrm{Y}$ or the relationship between arm length with service accuracy). While the multiple correlation test statistics are used to test the third hypothesis (X1, and $\mathrm{X} 2$ analysis with $\mathrm{Y}$ or the relationship between hand grip strength and arm length with service accuracy).

2). Research Hypothesis Testing The Relationship Between Hand Grip Strength and Service Accuracy (Correlation Analysis $\mathbf{X}_{1}$ to $\mathbf{Y}$ ).

To test whether there is a relationship between hand grip strength $\left(X_{1}\right)$ and service accuracy (Y) that is done by calculating $\mathrm{r}$ (correlation) through the formula:

$$
\begin{aligned}
& \begin{array}{|c|c|c|c|c|c|c|c|c|c|}
\hline \sum n & \sum \mathbf{X}_{1} & \sum \mathbf{X}_{2} & \sum Y & \sum \mathbf{X}_{1}{ }^{2} & \sum \mathbf{X}_{2}{ }^{2} & \sum \mathbf{Y}^{2} & \sum \mathbf{X}_{1} \mathbf{Y} & \sum \mathbf{X}_{2} \mathbf{Y} & \sum \mathbf{X}_{1} \mathbf{X}_{2} \\
\hline 40 & \mathbf{2 0 0 4} & \mathbf{2 0 0 3} & \mathbf{2 0 0 5} & \mathbf{1 0 4 2 7 8} & \mathbf{1 0 4 1 7 5} & \mathbf{1 0 4 4 2 9} & \mathbf{1 0 3 1 7 2} & \mathbf{1 0 2 9 4 2} & \mathbf{1 0 2 4 6 4} \\
\hline
\end{array} \\
& \Gamma x_{1} y=\frac{\mathrm{n} \Sigma \mathrm{X}_{1} \mathrm{y}-\left(\Sigma \mathrm{X}_{1}\right)(\Sigma \mathrm{y})}{\sqrt{\left\{\mathrm{n} \Sigma \mathrm{X}_{1}^{2}-\left(\Sigma \mathrm{X}_{1}\right)^{2}\right\}\left\{\mathrm{n} \Sigma \mathrm{y}^{2}-(\Sigma y)^{2}\right\}}} \\
& =\frac{40(103172)-(2004)(2005)}{\sqrt{\left(40 \times 104278-(2004)^{2}\right)\left(40 \times 104429-(2005)^{2}\right)}}=0.70
\end{aligned}
$$

The calculation results of the analysis of the relationship X1 with Y or between the strength of the hand grip with service accuracy, obtained the magnitude of the correlation coefficient $(r)=0.70$, based on the interpretation table correlation coefficient $r$ value this shows the level of a strong relationship between the strength of the hand grip with service accuracy. Furthermore, to state the size of the contribution of the variable X1 to $\mathrm{Y}$ can be determined by the formula of the determinant coefficient as follows: KP $=\mathrm{r} 2 \times 100 \%=0.702$ $\mathrm{x} 100 \%=49 \%$. Thus the magnitude of the contribution of hand grip strength to service accuracy is $49 \%$ and the remaining $51 \%$ is determined by other variables.

3). Research Hypothesis Testing Relationship Between Arm Length With Service Accuracy (Correlation Analysis $\mathbf{X}_{2}$ with $\mathbf{Y}$ )

\begin{tabular}{|c|c|c|c|c|c|c|c|c|c|}
\hline$\sum n$ & $\sum X_{1}$ & $\sum X_{2}$ & $\sum Y$ & $\sum X_{1}^{2}$ & $\sum X_{2}^{2}$ & $\sum Y^{2}$ & $\sum X_{1} Y$ & $\sum X_{2} Y$ & $\sum X_{1} X_{2}$ \\
\hline 40 & 2004 & $\mathbf{2 0 0 3}$ & $\mathbf{2 0 0 5}$ & $\mathbf{1 0 4 2 7 8}$ & $\mathbf{1 0 4 1 7 5}$ & $\mathbf{1 0 4 4 2 9}$ & $\mathbf{1 0 3 1 7 2}$ & $\mathbf{1 0 2 9 4 2}$ & $\mathbf{1 0 2 4 6 4}$ \\
\hline
\end{tabular}

Source: Appendix 4

$$
\begin{aligned}
\operatorname{rx}_{2} \mathrm{y} & =\frac{\mathrm{n} \Sigma \mathrm{X}_{2} \mathrm{y}-\left(\Sigma \mathrm{X}_{2}\right)(\Sigma \mathrm{y})}{\sqrt{\left\{\mathrm{nX}_{2}^{2}-\left(\Sigma \mathrm{X}_{2}\right)^{2}\right\} \hat{\left.\mathrm{n} \Sigma \mathrm{y}^{2}-(\Sigma y)^{2}\right\}}}} \\
& =\frac{40(102942)-(2003)(2005)}{\sqrt{\left(40 \times 104175-(2003)^{2}\right)\left(40 \times 104429-(2005)^{2}\right)}}=0.65
\end{aligned}
$$

The calculation results of the analysis of the relationship $\mathrm{X}_{2}$ with $\mathrm{Y}$ or between the length of the arm with service accuracy, obtained the magnitude of the correlation coefficient $=0.65$, based on the interpretation of the correlation coefficient table $r$ values this shows the level of a strong relationship between arm length with service accuracy. Furthermore, to state the size of the contribution of the variable $\mathrm{X}_{2}$ to $\mathrm{Y}$ can be determined by the formula of the determinant coefficient as follows: $\mathrm{KP}=\mathrm{r} 2 \times 100 \%=0.652 \times 100 \%=42.25 \%$. Thus the 
magnitude of the contribution of the variable arm length with service accuracy is $42.25 \%$ and the remaining $57.75 \%$ is determined by other variables.

4). Research Hypothesis Testing Relationship between the Strength of Hand Grips with Arm Length (Correlation Analysis $\mathbf{X}_{1}$ to $\mathbf{X}_{2}$ ).

To find out the magnitude of the relationship between $\mathrm{X}_{1}$ and $\mathrm{X}_{2}$, using the following formula:

\begin{tabular}{|c|c|c|c|c|c|c|c|c|c|}
\hline$\sum n$ & $\sum X_{1}$ & $\sum X_{2}$ & $\sum Y$ & $\sum X_{1}{ }^{2}$ & $\sum X_{2}^{2}$ & $\sum Y^{2}$ & $\sum X_{1} Y$ & $\sum X_{2} Y$ & $\sum X_{1} X_{2}$ \\
\hline 40 & 2004 & 2003 & 2005 & 104278 & 104175 & 104429 & 103172 & $\mathbf{1 0 2 9 4 2}$ & 102464 \\
\hline
\end{tabular}

Source: Appendix 4

$$
\begin{aligned}
\mathrm{rX}_{1} \mathrm{X}_{2} & =\frac{\mathrm{n} \Sigma \mathrm{X}_{1} \mathrm{X}_{2}-\left(\Sigma \mathrm{X}_{1}\right)\left(\Sigma \mathrm{X}_{2}\right)}{\sqrt{\left\{\mathrm{n} \Sigma \mathrm{X}_{1}^{2}-\left(\Sigma \mathrm{X}_{1}\right)^{2}\right\}\left\{\mathrm{n} \Sigma X_{2}^{2}-\left(\Sigma X_{2}\right)^{2}\right\}}} \\
& =\frac{40(102464)-(2004)(2003)}{\sqrt{\left(40 \times 104278-(2004)^{2}\right)\left(40 \times 104175-(2003)^{2}\right)}}=0.55
\end{aligned}
$$

The results of the analysis of the relationship between $X_{1}$ and $X_{2}$ or between the strength of the hand grip with the length of the arm, obtained the magnitude of the correlation coefficient $=0.55$. Based on the interpretation table correlation coefficient $r$ value this shows the level of a fairly strong relationship between the strength of the hand grip with the length of the arm. Furthermore, to state the size of the contribution of the variable $X_{1}$ to $X_{2}$ can be determined by the formula of the determinant coefficient as follows: KP $=\mathrm{r} 2 \times 100 \%=0.552$ $\mathrm{x} 100 \%=30.25 \%$. Thus the magnitude of the contribution of the variable strength of the hand grip with the length of the arm is $30.25 \%$ and the remaining $77.91 \%$ is determined by other variables.

\section{5). Multiple Correlation Analysis for $X 1$ and $X 2$ with $Y$}

The hypothesis that will be tested in this study is the relationship between hand grip strength and arm length together with the accuracy of field tennis services for seventh semester male students of the Department of Training in the Faculty of Sport Science, UNIMA. So to test this hypothesis by using the multiple correlation formula (double correlation), followed by calculating the significance of the multiple correlations with the formula:

$$
\mathrm{F}_{\text {count }}=\frac{\frac{R^{2}}{k}}{\frac{\left(1-R^{2}\right)}{n-k-1}}
$$

Calculation of research hypothesis testing is carried out with the following steps:

a. first step: determine the research hypothesis

Research hypotheses are:

Ho: there is no correlation between the strength of hand grip and arm length together with the accuracy of the tennis services of the eighth semester students of male students in the Department of Training in the Faculty of Sport Science, UNIMA.

Ha: there is a correlation between the strength of hand grip and arm length together with the accuracy of the tennis services of the eighth semester students of the students of the Department of Training in the Faculty of Sport Science, UNIMA.

b. Second step: determine the testing criteria. 
Testing criteria are:

Accept Ho if the observation $\square \mathrm{r}_{\text {table }}$ means that it is not significant

Reject Ho if observation $>r_{\text {table }}$ means significant.

Significance level $\square 0.05$ and known $=\mathrm{n}-\mathrm{k}-1=40-2-1=37$.

$$
\begin{aligned}
\mathrm{F}_{\text {table }} & =(\mathrm{dk}=\mathrm{k}),(\mathrm{dk}=\mathrm{n}-\mathrm{k}-1) \\
& =(\mathrm{dk}=2),(\mathrm{dk}=40-2-1) \\
& =2 \text { and } 37
\end{aligned}
$$

The way to find Ftable is number 2 as a numerator and 37 as a denominator. Thus a Ftabel of 3,225 (Interpolation) was found. (Appendix 9)

a. Third step: calculate Calculated through formulas:

$$
\mathrm{F}_{\text {count }}=\frac{\frac{R^{2}}{k}}{\frac{\left(1-R^{2}\right)}{n-k-1}}
$$

Before calculating $\mathrm{F}_{\text {count }}$ then first look for it $\mathrm{R}_{\mathrm{x} 1 \mathrm{x} 2 \mathrm{y}}$ or Multiple Correlation through the formula.

Table 10. Summary of Correlation Results

\begin{tabular}{|c|c|}
\hline Statistical Symbols & Statistical Value \\
\hline r $\times 1 . Y$ & 0.70 \\
\hline r $\times 2 . Y$ & 0.65 \\
\hline r x1. $\times 2$ & 0.55 \\
\hline
\end{tabular}

From the results of the correlation then entered in the multiple correlation formula (R) with the formula:

$$
\begin{aligned}
\operatorname{Rx}_{1} x_{2} \mathrm{y} & =\sqrt{\frac{r^{2} x_{1} y+r^{2} x_{2} y-2\left(r x_{1} y\right)\left(r x_{2} y\right)\left(r x_{1} x_{2}\right)}{1-r^{2} x_{1} x_{2}}} \\
& =\sqrt{\frac{0.4900+0.4225-2(0.70)(0.65)(0.55)}{1-(0.55)^{2}}} \\
& =\sqrt{\frac{0.9125-0.5005}{0.6975}} \\
& =\sqrt{\frac{0.4120}{0.6975}} \\
& =\sqrt{0.590681003} \\
& =0.768557742 \\
& =0.77 \text { (rounded off) }
\end{aligned}
$$

The simultaneous relationship between hand grip strength and arm length with the accuracy of the eighth semester student field tennis services of the Department of Sport Training of the Faculty of Sport Sciences UNIMA is relatively strong. The simultaneous contribution of variables $\mathrm{X}_{1}$ and $\mathrm{X}_{2}$ to $\mathrm{Y}=\mathrm{r} 2 \times 100 \%=0.772 \times 100 \%=59.29 \%$ and the remaining $40.71 \%$ is determined by other variables.

Next to test the significance of the proposed hypothesis through the formula: 


$$
\begin{aligned}
\mathrm{F}_{\text {count }} & =\frac{\frac{R^{2}}{k}}{\frac{\left(1-R^{2}\right)}{n-k-1}} \\
& =\frac{0.77^{2} / 2}{\left(1-0.77^{2}\right) / 40-2-1} \\
& =\frac{0.5929 / 2}{(1-0.5929) / 37} \\
& =\frac{0.29645}{0.4071 / 37} \\
& =\frac{0.29645}{0.011002702} \\
& =26.94338173 \\
& =26.94 \text { (rounded off) }
\end{aligned}
$$

d. Step four: conclude the calculation results.

From the results of testing the research hypothesis obtained Fcount $=26.94$ with a level of confidence $\square 0.05$ and $\mathrm{dk}=\mathrm{n}-\mathrm{k}-1=40-2-1=37$, so it was found Ftable $=3.225$. So $F_{\text {count }}$ is greater than $F \neg_{\text {table }}$, i.e. $F_{\text {count }}=26.94>F_{\text {table }}=3.225$. In accordance with the testing criteria if $\mathrm{F}_{\text {count }}>\mathrm{F}_{\text {table }}$ then $\mathrm{Ho}$ is rejected, which means accept $\mathrm{Ha}$ or a hypothesis stating there is a correlation between the strength of hand grip and arm length together with the accuracy of field tennis services in the eighth semester students of the Department of Coaching Education at the Faculty of Sports, UNIMA.

Based on the test results described earlier it is proven that there is a significant relationship between the strength of hand grip and arm length together with the accuracy of the tennis services of the eighth semester male student tennis department of the Department of Training in the Faculty of Sport Science, UNIMA. The full description is as follows:

1. There is a Relationship between Hand Grip Strength $\left(X_{1}\right)$ with Service Accuracy (Y). Based on the results of testing analysis between the strength of the hand grip with service accuracy, the correlation coefficient $(r)=0.70$, based on the interpretation coefficient table $\mathrm{r}$ correlation value shows that there is a strong relationship between hand grip strength and service accuracy. Furthermore, the contribution of hand grip strength to service accuracy is $49 \%$ and the remaining $51 \%$ is determined by other variables.

2. There is a Relationship between Arm Length $\left(\mathrm{X}_{2}\right)$ and Service Accuracy (Y)

Based on the calculation results of the analysis of the relationship $\mathrm{X}_{2}$ with $\mathrm{Y}$ or between the length of the arm with the accuracy of service, obtained the magnitude of the correlation coefficient $=0.65$, based on the interpretation coefficient table correlation value of $r$ this shows the level of a strong relationship between arm length with service accuracy. Furthermore, to state the size of the contribution of the $\mathrm{X}_{2}$ variable to $\mathrm{Y}$ or the arm length variable with service accuracy is $42.25 \%$ and the remaining $57.75 \%$ is determined by other variables.

3. There is a Simultaneous Relationship between the Strength of Hand Grips and Arm Length with Accuracy in Field Tennis Services for $7^{\text {th }}$ Semester Male Students Department of Training in the Faculty of Sport Science UNIMA 
The simultaneous relationship between hand grip strength and arm length with accuracy of field tennis services for eighth semester students of the Department of Sport Training of the Faculty of Sport Science UNIMA is relatively strong, where a correlation $r$ value of 0.77 is obtained. The simultaneous contribution of variables $\mathrm{X}_{1}$ and $\mathrm{X}_{2}$ to $\mathrm{Y}=\mathrm{r} 2 \times 100 \%=0.772$ $\mathrm{x} 100 \%=59.29 \%$ and the remaining $40.71 \%$ is determined by other variables.

The results of the calculation of the analysis of the relationship $\mathrm{X}_{1}$ and $\mathrm{X}_{2}$ together with (Y), showed the magnitude of $\mathrm{F}_{\text {count }}=26.94$ with a level of confidence $\square 0.05$ and $\mathrm{dk}=\mathrm{n}-\mathrm{k}$ $-1=40-2-1=37$, so it was found $F_{\text {table }}=3.225$. So $F_{\text {count }}$ is greater than $F_{\neg t a b l e}$, i.e. $F_{\text {count }}=$ 26.94> $\mathrm{F}_{\text {table }}=3.225$. In accordance with the testing criteria if $F_{\text {coun }}>F_{\text {table }}$ then Ho is rejected, which means accept Ha or a hypothesis stating there is a correlation between the strength of hand grip and arm length together with the accuracy of field tennis services in the eighth semester students of the Department of Coaching Education at the Faculty of Sports, UNIMA.

The results of the discussion above show that the strength of the hand grip and arm length are very important in determining the accuracy of field tennis services for seventh semester male students of the Department of Training in the Faculty of Sport Science, UNIMA. Students who have the strength of hand grip and arm length in servicing in playing tennis will be better prepared and easier to do service accuracy.

Thus the conclusion in this study is that there is a significant relationship between the strength of hand grip and arm length together with the accuracy of field tennis services for seventh semester male students at the Department of Coaching Education at the Faculty of Sport Science, UNIMA.

\section{Conclusion}

There is a significant relationship between hand grip strength and service accuracy in semester VII male students of the Department of Coaching Education. There is a meaningful relationship between arm length and correct service in $7^{\text {th }}$ semester male students in Department of Coaching Education. There is a meaningful relationship between hand grip strength and arm length together with the accuracy of service to seventh semester male students of the Department of Training Education.

\section{References}

Bompa O. Tudor. (1983) Theory and Methodology of Training, Kendall/Hunt Publishing. Company. Dubuque. Lowa.

Chu A. Donald. (2000). Tenis Tenaga. Terjemahan Siregar Rusi. PT Raja Grafindo Persada. Jakarta.

Gautschi Marcel. (1988). Efektifitas Tenis Metode Berlatih Bermain dan Berprestasi. Dahara Prize. Semarang.

Gunarsa D. (1989). Singgih, Psikologi Olahraga. PT BPK Gunung Mulia. Jakarta.

Harsono. (1988). Coaching dan Aspek-Aspek Psikologi dalam Olahraga. P2LPTK. Jakarta.

Harsono. (1986). Ilmu Coaching. PIO KONI Pusat. Jakrta.

Lerdner Rex. (2003). Pedoman Lengkap Bermain Tenis Strategi dan Teknik Yang Akurat. Dahara Prize. Semarang.

Midgley Rud. (1996). Ensiklopedi Olahraga. Dahara Prize. Semarang,.

Mutalib Peni. (1977). Mengukur Kemampuan Fisik Pengolahraga Secara Sederhana. Arcan. Jakarta. 
Novianti, D. (2020). Improvement of Physical, Honesty, Discipline and Cooperation in Class IV Elementary School Students through Circuit Training Learning Model Budapest International Research and Critics in Linguistics and Education (BirLE) Journal. p. 244-250.

Pearce Evelins.( 1990) Anatomi dan Fisiologi Untuk Para Medis. Gramedia Pustaka Utama. Jakarta,.

Raven Chr P.( 1997).Atlas Anatomi. Djambatan. Jakarta.

Riduan. (2010). Metode dan Teknik Menyusun Tesis. Tarsito Alfabeta. Bandung.

Sajoto Mochamad.( 1988) Pembinaan Kondisi Fisik Dalam Olahraga. DEPDIKBUD DIKTI P2LPTK. Jakarta.

Sekretaris Umum PP.PBVSI. (1995). Metodologi Pelatihan. Jakarta.

- Siregar Syofian. (2011). Statistika Deskriptif Untuk Peneletian. Rajawali Pers Devisi Buku Perguruan Tinggi PT Raja Gratindo Persada. Jakarta.

Soediharso. (1974). Tenis Bagi Pembina Olahraga. Dirjen Olahraga dan Pemuda. Depdikbud. Jakarta.

Sudjana. (1992). Metode Statistika. Tarsito. Bandung.

Suharsimi Arikunto. (2010). Produser Penelitian. Rineka Cipta. Jakarta.

Syafuddin.( 1992). Anatomi Fisiologi Untuk Perawat, Buku Kedokteran. Jakarta.

Witarsa. (2002).Latihan Kondisi Fisik. Penataran Wasit dan Latihan Panahan Sejawa Barat. Bandung. 